\title{
DPPH (1,1-Diphenyl-2-Picrylhydrazyl) Radical Scavenging Activity of Flavonoids Obtained from Some Medicinal Plants
}

\author{
Masafumi OKawa, ${ }^{a}$ Junei KInJo, ${ }^{b}$ Toshihiro Nohara, ${ }^{* a}$ and Masateru ONO ${ }^{c}$ \\ Faculty of Pharmaceutical Sciences, Kumamoto University, ${ }^{a}$ 5-1 Oe-Honmachi, Kumamoto 862-0973, Japan, Faculty of \\ Pharmaceutical Sciences, Fukuoka University, ${ }^{b}$ 8-19-1 Nanakuma, Fukuoka 814-0180, Japan, and School of Agriculture, \\ Kyushu Tokai University, ${ }^{c} 5435$ Choyo, Aso, Kumamoto 869-1404, Japan. \\ Received March 5, 2001; accepted July 21, 2001
}

\begin{abstract}
A reactive oxygen species has been implicated in a range of human pathological diseases such as atherosclerosis and certain cancers. Flavonoids are reported to exhibit various biological activities, including antioxidative and free radical scavenging activities. Several flavonoids obtained from barley leaves, soybean and some medicinal plants, Silybum marianum, Sophorae Flos, Cinnamon, Ephedrae Herba and Scutellariae Radix, were tested for their DPPH (1,1-diphenyl-2-picrylhydrazyl) radical scavenging activity. The structure-activity relationships suggested that not only the numbers of hydroxy group but also the position of hydroxy group might be important for mediating potent activity.
\end{abstract}

Key words flavonoid; flavone; flavonol; DPPH radical scavenging activity; antioxidative activity; ESR

Flavonoids are a group of polyphenolic compounds, which have the diphenylpropane $\left(\mathrm{C}_{6}-\mathrm{C}_{3}-\mathrm{C}_{6}\right)$ skeleton, ubiquitously found in fruits and vegetables. The flavonoid family includes flavones, flavonols, flavanones, flavanonols, flavans, flavanols, leucoanthocyanidins, anthocyanidins, aurones, chalcones, and isofavones. The structural difference in each flavonoid family results from the variation in the number and arrangement of the hydroxyl groups and the extent of glycosylation of these groups. ${ }^{1)}$

Epidemiological studies suggest that the consumption of flavonoids is effective in lowering the risk of coronary heart disease. ${ }^{2-4)}$ In addition, the flavonoids exhibit a wide range of biological activities, including anticarcinogenic, antiinflammatory, antiradical, and antioxidant actions. Especially, they may exert antioxidative effects as free radical scavengers, hydrogen-donating compounds, singlet oxygen quenchers, and metal ion chelators, properties attributed to the phenolic hydroxyl groups attached to the ring structures.

Nowadays, it is reported that a reactive oxygen species (ROS) is implicated in a wide range of human diseases such as atherosclerosis and certain cancers. When an imbalance between ROS generation and antioxidants occurs, oxidative damage will spread over all the cell targets (DNA, lipids, proteins). ${ }^{5}$ Antioxidants in foods and medicinal plants (or herbs) have attracted interest in recent years. Flavonoids given as biological substances in foodstuffs may contribute to the prevention of diseases, although they do not have strong biological activities. ${ }^{6-8)}$

In the preceding paper, ${ }^{9)}$ we reported the antioxidative effects of flavon $C$-glycosides isolated from young green barley (Hordeum vulgare var. nudum) leaves. Herein, we present antioxidative effects of various types of flavonoids obtained from some medicinal plants, using DPPH (1,1-diphenyl-2picrylhydrazyl) radical scavenging activity by ESR.

\section{MATERIALS AND METHODS}

Flavonoids Flavone $C$-glycosides, isovitexin (1), saponarin (2), isovitexin $4^{\prime}, 7$-diglucoside (3), isovitexin 7-rhamnosylglucoside (4), 6"'-sinapoylsaponarin (5), 6"'--feruloyl- saponarin (6) and 4' -glycosyl-6"'-sinapoylsaponarin (7) were isolated from young barley leaves. ${ }^{9)}$ A flavone, baicalin ${ }^{10)}(\mathbf{8})$ was obtained from Scutellariae Radix (Scutellaria baicalensis). Flavonols, quercetin (9), kaempferol (10) and rutin (11) were obtained from Sophorae Flos (Sophora japonica). ${ }^{11)}$ Flavanols, gallocatechin (12) was obtained from Ephedrae Herba (Ephedra sinica) and epicatechin (13) and their polymers (14-16) were from cinnamon (Cinnamomum cassia) ${ }^{12)}$ Isoflavones ${ }^{13)}$ daidzin (17), genistin (18) and glycitin (19), and their metabolites ${ }^{14)}(\mathbf{2 0}-\mathbf{2 5})$ were obtained from soybeen (Glycine max). Flavonolignans, silybin (26) and silychristin (27) were isolated from a silymarin group (Silybum marianum) ${ }^{15)}$ (Fig. 1).

DPPH Radical Scavenging Activity by ESR The DPPH radical scavenging activity of a test sample was estimated by ESR according to the method of Tateyama et al. ${ }^{16)}$ Various concentrations of these flavonoids in DMSO were added to $150 \mu \mathrm{M}$ DPPH radical in ethanol and mixed for $10 \mathrm{~s}$. After further mixing for $60 \mathrm{~s}$, ESR spectra were recorded using a JEOL at $100 \mathrm{KHz}$ field modulation frequency and $1 \mathrm{G}$ modulation amplitude at an output power of $8 \mathrm{~mW}$. Mn(II) was used as standard sample. All experiments were carried out at room temperature $\left(25^{\circ} \mathrm{C}\right)$. DPPH radical reducing activity of the test sample was expressed as $I=\left[\left(A_{0} / A_{\mathrm{X}}\right) / A_{0}\right]$ $\times 100 \%$, where $A_{0}$ and $A_{\mathrm{X}}$ were the relative heights of each radical against the standard signal intensity of the manganese oxide marker in a reaction mixture and the test sample, respectively. The results were indicated as the concentration required to effect a $50 \%$ inhibition of decreasing signal peak height $\left(\mathrm{IC}_{50}\right)$ by ESR.

\section{RESULTS AND DISCUSSION}

Seven flavones 1-7 isolated from barley leaves showed similar results (Table 1 ) to those obtained from a previous investigation $^{9)}$ by the colorimetric method. Since 5-7 (5: $\left.\mathrm{IC}_{50}=21.1 \mu \mathrm{M}, \mathbf{6}: \mathrm{IC}_{50}=51.1 \mu \mathrm{M}, 7: \mathrm{IC}_{50}=25.5 \mu \mathrm{M}\right)$ exhibited stronger DPPH radical scavenging effect than did 1-4 (1: $\mathrm{IC}_{50}>1000 \mu \mathrm{M}, 2: \mathrm{IC}_{50}=211 \mu \mathrm{m}, 3: \mathrm{IC}_{50}=291 \mu \mathrm{M}, 4: \mathrm{IC}_{50}=$ $299 \mu \mathrm{M})$, the importance of sinapoyl and feruloyl groups for 

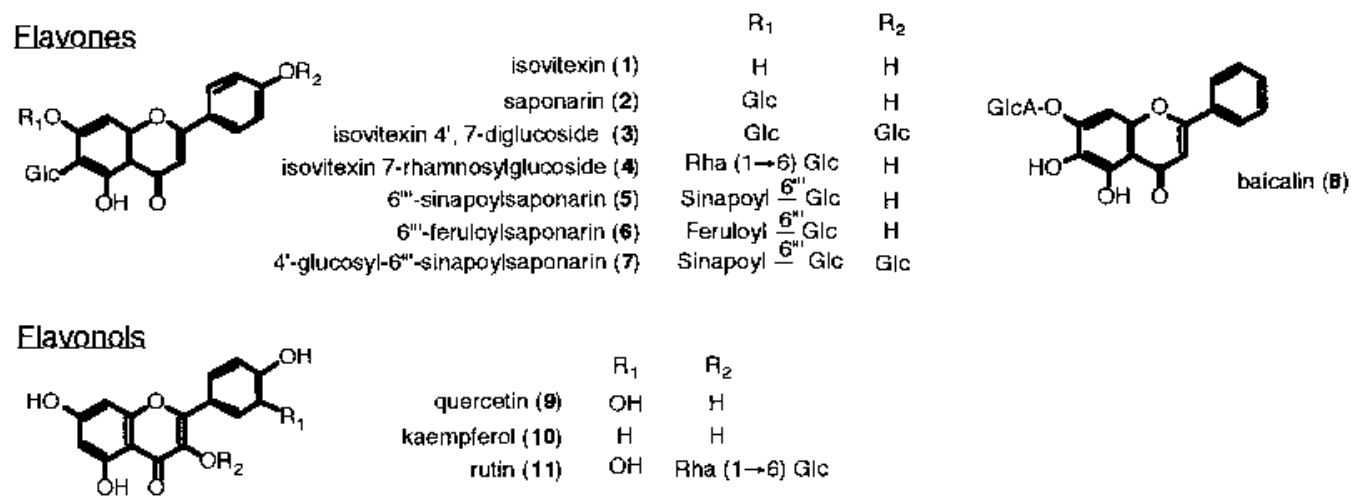

Flavanols
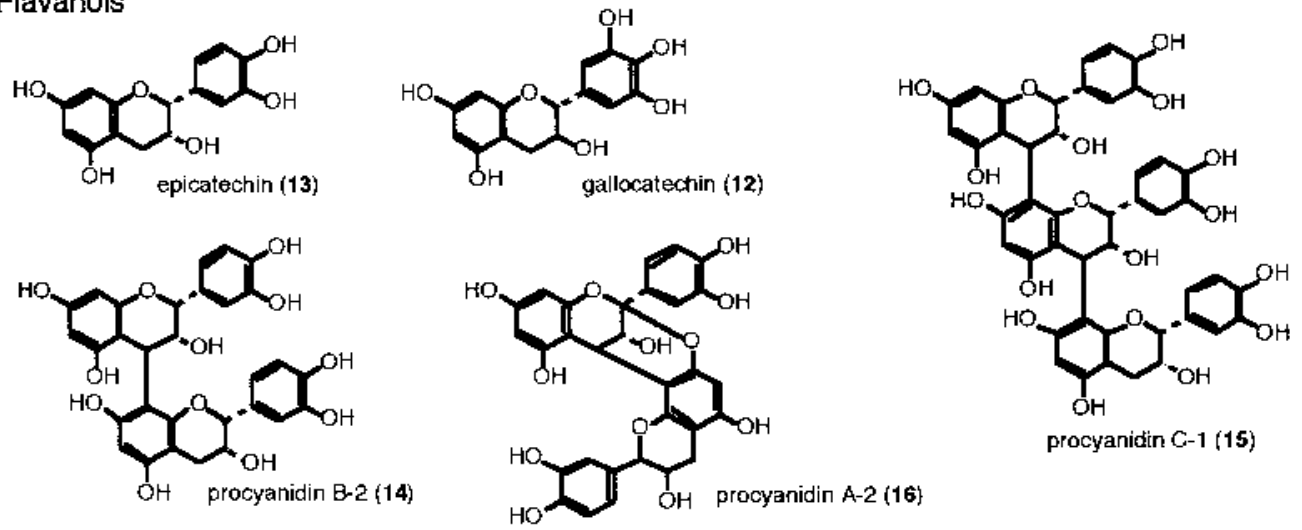

1soflavones
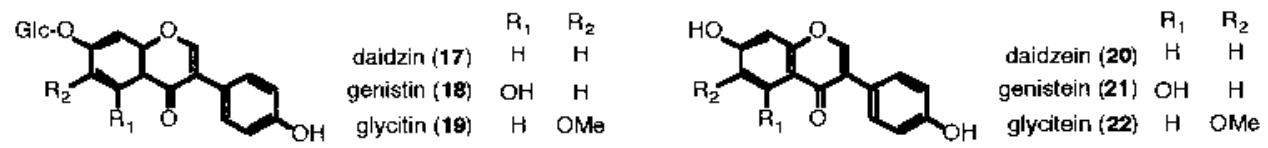

Isoflavanones

Isoflavan
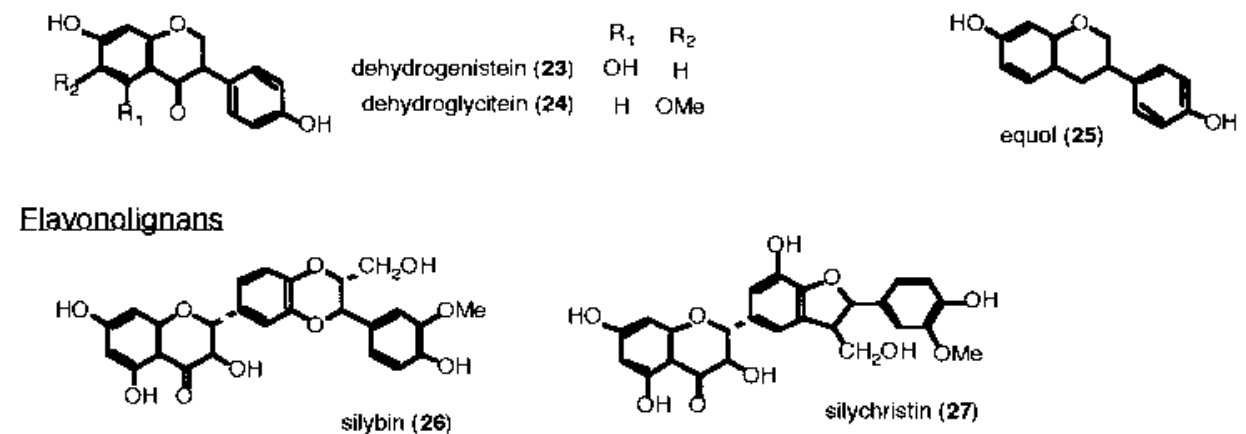

Fig. 1

the DPPH radical reducing activity was confirmed. Furthermore, baicalin (8) having 5,6,7-trihydroxy group showed the strongest activity in the tested flavones $(\mathbf{1}-\mathbf{8})$.

Despite having three hydroxy groups, isovitexin (1) did not exhibit any activity similarly to the former experiment. ${ }^{9)}$ Since baicalin (8) has no hydroxy group on the B-ring, the hydroxy group at C-4' may not contribute to scavenge for DPPH radical. Probably, the hydroxy group at C- 6 of 8 plays a role in scavenging DPPH radical.

Furthermore, nineteen flavonoids (8-27) belonging to six flavonoid classes (flavonol, flavanol, isoflavone, isoflavanone, isoflavan and flavonolignan) were prepared from some medicinal plants, Sophora japonica, Ephedra sinica, Cinnamomum cassia, Glycine max and Silybum marianum, and their DPPH radical reducing activity was compared. The results of DPPH radical reducing activity were shown in Table 1.

The structure of flavonolignans, silybin (26) and silychristin (27), was constitued of a flavanonol unit and a phenylpropanol unit in analog with 5-7 which showed potent activity. However, the activity of $\mathbf{2 6}$ and $\mathbf{2 7}$ was much weaker than 5-7. Especially, 26 did not show any activity, although silychristin (27) having a hydroxy group at C-3' 
Table 1. Inhibitory Activity of Various Types of Flavonoids on DPPH Radical

\begin{tabular}{|c|c|c|}
\hline Compounds & No. & $\mathrm{IC}_{50}(\mu \mathrm{M})$ \\
\hline \multicolumn{3}{|l|}{$\begin{array}{l}\text { Flavones } \\
\text { (Glycosides) }\end{array}$} \\
\hline Isovitexin & 1 & $>1000$ \\
\hline Saponarin & 2 & 211 \\
\hline Isovitexin 4',7-diglucoside & 3 & 291 \\
\hline Isovitexin 7-rhamnosylglucoside & 4 & 299 \\
\hline 6"'-Sinapoylsaponarin & 5 & 21.1 \\
\hline $6^{\prime \prime \prime}$-Feruloylsaponarin & 6 & 51.1 \\
\hline 4'-Glucosyl-6"'-sinapoylsaponarin & 7 & 25.5 \\
\hline Baicalin & 8 & 15.5 \\
\hline \multicolumn{3}{|l|}{$\begin{array}{l}\text { Flavonols } \\
\text { (Aglycones) }\end{array}$} \\
\hline Quercetin & 9 & 8.9 \\
\hline $\begin{array}{l}\text { Kaempferol } \\
\text { (Glycoside) }\end{array}$ & 10 & 41.2 \\
\hline Rutin & 11 & 11.1 \\
\hline \multicolumn{3}{|l|}{ Flavanols } \\
\hline Gallocatechin & 12 & 6.9 \\
\hline Epicatechin & 13 & 11.7 \\
\hline Procyanidin B-2 & 14 & 11.7 \\
\hline Procyanidin C-1 & 15 & 7.3 \\
\hline Procyanidin A-2 & 16 & 8.8 \\
\hline \multicolumn{3}{|l|}{ Isoflavones } \\
\hline Daidzin & 17 & 642 \\
\hline Genistin & 18 & 265 \\
\hline Glycitin & 19 & 743 \\
\hline \multicolumn{3}{|l|}{ (Aglycones) } \\
\hline Daidzein & 20 & 532 \\
\hline Genistein & 21 & 1000 \\
\hline Glycitein & 22 & 612 \\
\hline \multicolumn{3}{|l|}{ Isoflavanones } \\
\hline Dihydrogenistein & 23 & $>1000$ \\
\hline Dihydroglycitein & 24 & 311 \\
\hline \multicolumn{3}{|l|}{ Isoflavan } \\
\hline Equol & 25 & 188 \\
\hline \multicolumn{3}{|l|}{ Flavonolignans } \\
\hline Silybin & 26 & $>1000$ \\
\hline Silychristin & 27 & 274 \\
\hline
\end{tabular}

showed moderate activity. The DPPH radical reducing activity of $\mathbf{2 6}$ and $\mathbf{2 7}$ was weaker than that of furulic acid itself. Accordingly, $\alpha, \beta$-unsaturated ketone might participate in enhancing activity.

In the flavonol class, quercetin (9) having a 3,5,7,3',4'pentahydroxy group showed stronger DPPH radical scavenging activity than kaempferol (10) which has 3,5,7,4'-tetrahydroxy group. Therefore, the dihydroxy group at C-3', 4' (catechol) in the B-ring of flavonol skeleton might enhance DPPH radical reducing activity. Since the 3-O-glycoside (11) of 9 exhibited similar activity with $\mathbf{9}$, the hydroxy group at C-3 may not contribute to scavenge for DPPH radical.

In a flavanol group, the order of reducing activity was gallocatechin (12) $\geq$ procyanidin C-1 (15) $\geq$ procyanidin A-2

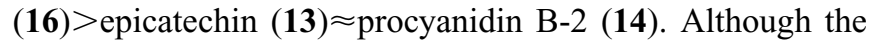
trimer (15) has numerous hudroxy groups, gallocatechin (12) was slightly effective than $\mathbf{1 5}$. Similarly, the activity of dimer (14) was equal to that of the monomer (13). Finally, procyanidin A-2 (16) was more potent than 14, despite the lack of a hydroxy group. Therefore, the number of hydroxy groups seemed not to be always important. On the other hand, it is almost certain that the position of hydroxy group (catechol or pyrogallol) was more important than the number of hydroxy groups.

In the case of isofavone, isoflavanone and isoflavan family (17-25), a isoflavan (25) exhibited stronger activity than the others. Successively, genistin (18) showed stronger activity, although the other isoflavone glycosides were much less potent. Generally, the DPPH radical reducing activity of isoflavonoid (isofavone, isoflavanone and isoflavan) was weaker than those of the other class of flavonoid (flavone, flavonol and flavanol).

The flavonoids $(\mathbf{9}, \mathbf{1 1}-\mathbf{1 6})$ which showed potent activity have a catechol unit in their structure and $\mathbf{8}$ has a similar feature carrying an adjacent dihydroxy group (C-5, C-6) in the A ring. On the other hand, less potent compounds 26 and 27 have a guaiacyl group instead of a catechol group. Therefore, the significance of catechol group on DPPH radical reducing activity was thus confirmed. ${ }^{17,18)}$ However, the number of hydroxy groups was not to be always important when compared with the activity of $\mathbf{1 2}$ and $\mathbf{1 5}$. The position of hydroxy group might be more important for mediating potent DPPH reducing activity.

Various antioxidants are contained in most foods and medicinal plants. They are polyphenols, vitamins, carotenoids, and flavonoids in vegetables and fruits. The recent study on antioxidative substances in foods and medicinal plants is a comparatively new province. The digestion, absorption, biological activity and metabolic pathway in each food and medicinal plant is still complicated because a number of substances are included in various proportions in one plant. Meanwhile, their safety has been established to a certain extent for empirical and traditional use from ancient times. Therefore, the study of antioxidative substances in foods and medicinal plants will become important, and such antioxidative substances might be applied for treatment and prevention of human diseases.

Acknowledgement We are grateful to Dr. G. Nonaka (Usaien Pharmaceutical Co.) for his helpful suggestion.

\section{REFERENCES AND NOTES}

1) Rice-Evans C. A., Miller N. J., Paganga G., Free Radical Biol. Med., 20, 933-956 (1996).

2) Rice-Evans C. A., Miller N. J., Biochem. Soc. Trans., 24, 790-795 (1996).

3) Block G., Nutr. Rev., 50, 207-213 (1992).

4) Hollman P. C. H., Hertog M. G. L., Katan M. B., Biochem. Soc. Trans., 24, 785-789 (1996).

5) Cook N. S., Samman S., J. Nutr. Biochem., 7, 66-76 (1996).

6) Halliwell B., Aeschbach R., Loliger J., Aruoma O. I., Fd. Chem. Toxicol., 33, 601-617 (1995).

7) Manach C., Regerat F., Texier O., Agullo G., Demingne C., Remesy C., Nutr. Res., 16, 517-544 (1996).

8) Hackett A. M. (Cody V., Middleton E., Harborne J. B. eds.), Plant Flavonoids in Biology and Medicine, Biochemical Pharmcological and Structure Activity Relationships, 177, New York Alan R. Liss Inc., (1986).

9) Okawa M., Kinjo J., Hagiwara Y., Hagiwara H., Ueyama H., Nakamura K., Ishikawa R., Ono M., Nohara T., Chem. Pharm. Bull., 46, 1887-1890 (1998)

10) Ishimaru K., Nishikawa K., Omoto T., Asai I., Yoshihira K., Shimomura K., Phytochemistry, 40, 279-281 (1995).

11) Liu I. M., Sheu S. J., Am. J. Chin. Med., 17, 179-187 (1989).

12) Nonaka G., Morimoto S., Kinjo J., Nohara T., Nishioka I., Chem. Pharm. Bull., 35, 149-155 (1987). 
13) Song T., Barua K., Buseman G., Murphy P. A., Am. J. Clin. Nutr., 68, 1474S-1479S (1998).

14) Kinjo J., Matsushita S., Shinohara M., Hirakawa T., Okawa M., Nohara T., Uchiyama S., Ueno T., Sogawa Y., Abstracts of Papers, The 46th Annual Meeting of the Japanese Society of Pharmacognosy, Osaka, Sep. 1999, p. 158.

15) Bosisio E., Benelli C., Pirola O., Pharmacol. Res., 25, 147-154
(1992).

16) Tateyama C., Ohta M., Uchiyama T., Nippon Kagaku Kougaku Kaishi, 44, 640-646 (1997).

17) Kondo K., Kurihara M., Miyata N., Suzuki T., Toyoda M., Arch. Biochem. Biophys., 362, 79-86 (1999).

18) Senba Y., Kishishita T., Saito K., Yoshioka H., Yoshioka H. T., Chem. Pharm. Bull., 47, 1369-1374 (1999). 